\title{
DICE simplified
}

\author{
Masako Ikefuji • Roger J. A. Laeven . \\ Jan R. Magnus • Chris Muris
}

Received: date / Accepted: date

\begin{abstract}
We analyze Nordhaus' DICE model and show that the temperature and $\mathrm{CO}_{2}$ equations are needlessly complicated and can be simplified without loss of essence. In addition, we argue that the damage function can be altered in such a way that it lends itself to experiments involving extreme risk. We conclude that, within the philosophy of the DICE model, significant simplifications can be made which make the model more transparent, more robust, and easier to apply.
\end{abstract}

Keywords DICE model · Climate change - Integrated assessment model · Optimal policy · Damage function

Mathematics Subject Classification (2010) 93A30 - 91B76

This research was funded in part by the Japan Society for the Promotion of Science (JSPS), KAKENHI Grant JP19K01669 (Ikefuji), and the Netherlands Organization for Scientific Research (NWO) under grant Vidi-2009 (Laeven).

Masako Ikefuji

Faculty of Humanities and Social Sciences, University of Tsukuba

and ISER, Osaka University, Japan

E-mail: ikefuki.masako.gn@u.tsukuba.ac.jp

Roger J. A. Laeven

Amsterdam School of Economics, University of Amsterdam, CentER, and EURANDOM, The Netherlands

E-mail: R.J.A.Laeven@uva.nl

Jan R. Magnus

Department of Econometrics and Data Science, Vrije Universiteit Amsterdam, and Tinbergen Institute, The Netherlands

E-mail: jan@janmagnus.nl

Chris Muris

Department of Economics, McMaster University, Canada

E-mail: muerisc@mcmaster.ca 
Entia non sunt multiplicanda praeter necessitatem William of Ockham, 1285(?)-1347

\section{Introduction}

This paper provides a critical assessment of the 2016 DICE (Dynamic Integrated model of Climate and the Economy) model originally developed by Nordhaus [17], but since then continuously updated and altered (see [22]). Integrated assessment models (IAMs) are being used extensively for the analysis of climate change policy and DICE has played an important part in projecting greenhouse gas emissions and temperature under various social and economic scenarios.

The assumptions and functional forms used in DICE have been under considerable scrutiny and criticism. Among others, $[23,24,8,9]$ argue that IAMs should be made simpler and more transparent, and an editorial in Nature Climate Change [6] supports this view. DICE is complex, because it employs an equation for radiative forcing, two equations (a two-box model) for the climate system, and a three-reservoir model for the carbon cycle. Some of the other IAMs (for example, the FUND and PAGE models) are somewhat less complex, as they employ a single-equation climate model (see [3]).

The purpose of this paper is to show that the temperature and $\mathrm{CO}_{2}$ equations in DICE are needlessly complicated and can be much simplified. The technical reason why this simplification is possible lies in the fact that the matrix connecting the two dynamic equations describing temperature in the DICE model has one eigenvalue close to one, and that the matrix connecting the three equations describing $\mathrm{CO}_{2}$ has one exact and one approximate unit eigenvalue. We derive the equivalent equations after differencing out the auxiliary variables and we provide simplifications.

In response to criticism that IAMs give the impression of being 'black boxes' ([6]), one approach is to propose significant changes to IAMs such as DICE, leading to simpler IAMs with an analytical formula for the optimal carbon price; see $[10,29,25,30,5,31]$. Among studies employing closed-form IAMs, the specifications of the climate system and the carbon cycle vary. Golosov et al. [10] specify a twoand-a-half-box carbon system consisting of a permanent component (about $20 \%$ of carbon) and a transient component. The climate system is omitted. Under assumptions such as logarithmic utility, Cobb-Douglas technology, constant saving rate, and full depreciation of capital, they derive the optimal-tax formula analytically. Rezai and Van der Ploeg [25] employ a similar two-box carbon system. They allow for a lag between temperature and atmospheric carbon in addition to more general functional forms than those assumed by [10], and they derive a simple rule for the optimal carbon price.

The omission of the climate system in [10] is justified by recent findings in climate science that the climate response to a $\mathrm{CO}_{2}$ emission is nearly instantaneous and remains almost constant over time; see $[27,26]$. Based on the same findings, Dietz and Venmans [5] assume that the global mean temperature is linearly proportional to cumulative $\mathrm{CO}_{2}$ emissions, in contrast to the large thermal inertia of the climate system assumed in DICE and [16]. These differences may lead to different optimal transition paths of temperature. Thus, the optimal carbon price follows Hotelling's rule in [5], whereas it grows more slowly in [16]. 
Our approach is to stay as close as possible to Nordhaus' DICE model, but to simplify it using Ockham's razor quoted above ('more things should not be used than are necessary'). A complex model is not necessarily better than a simple model. Leaving things out is arguably more difficult and more important than putting things in. Many statisticians believe that a more complex model will reduce the bias and increase the variance, but this is only half true. A more complex model does indeed increase the variance, but it does not necessarily reduce the bias (see [4]). Hence, simplicity matters given the large uncertainties in the exogenous variables (such as population and technical knowledge) and the parameters.

We shall not propose a completely different climate system nor do we examine climate sensitivity in the DICE model. Our purpose is more modest. We shall show that the temperature and $\mathrm{CO}_{2}$ equations in the DICE model are needlessly complicated and that they can be much simplified. We also argue that the specification of the damage function can be altered in such a way that it lends itself to experiments involving extreme risk. Finally, we briefly discuss the assumption in DICE that the abatement fraction for $\mathrm{CO}_{2}$ is allowed to become larger than one, which implies that emissions can become negative.

In Sect. 2 we present the Nordhaus DICE 2016R model. In Sect. 3 and 4 we discuss and simplify the DICE equations for temperature and $\mathrm{CO}_{2}$ concentration. In Sect. 5 we provide an alternative to the DICE damage function. Sect. 6 presents and optimizes the S-DICE (simplified DICE) model and concludes.

\section{Nordhaus' DICE 2016R model}

The following equations are the equations from the beta version of DICE-2016R [20,21], a version with the identification DICE-2016R-091916ap.gms. A number of equations are redundant and have been deleted. A new variable $\omega_{t}$ has been introduced, some equations have been combined, and the equations have been reordered; see [14] for the details. Still, this is precisely the same model as Nordhaus' 2016R model.

Everybody works. In period $t$, the labor force $L_{t}$ together with the capital stock $K_{t}$ generate GDP $Y_{t}$ through a Cobb-Douglas production function

$$
Y_{t}=A_{t} K_{t}^{\gamma} L_{t}^{1-\gamma} \quad(0<\gamma<1)
$$

where $A_{t}$ represents technological efficiency and $\gamma$ is the elasticity of capital. Capital is accumulated through

$$
K_{t+1}=(1-\delta) K_{t}+I_{t} \quad(0<\delta<1),
$$

where $I_{t}$ denotes investment and $\delta$ is the depreciation rate of capital.

Carbon dioxide $\left(\mathrm{CO}_{2}\right)$ emissions consist of industrial and non-industrial ('landuse') emissions. We denote the latter type by $E_{t}^{0}$ and consider it to be exogenous to our model. Total $\mathrm{CO}_{2}$ emissions $E_{t}$ are then given by

$$
E_{t}=\sigma_{t}\left(1-\mu_{t}\right) Y_{t}+E_{t}^{0}
$$

where $\sigma_{t}$ denotes the emissions-to-output ratio for $\mathrm{CO}_{2}$ and $\mu_{t}$ is the abatement fraction for $\mathrm{CO}_{2}$. The associated $\mathrm{CO}_{2}$ concentration increase $M_{t}$ in the atmosphere 
(GtC from 1750) accumulates through

$$
\begin{aligned}
M_{t+1} & =\left(1-b_{0}\right) M_{t}+b_{1} X_{1, t}+E_{t}, \\
X_{1, t+1} & =b_{0} M_{t}+\left(1-b_{1}-b_{3}\right) X_{1, t}+b_{2} X_{2, t}, \\
X_{2, t+1} & =b_{3} X_{1, t}+\left(1-b_{2}\right) X_{2, t},
\end{aligned}
$$

where $X_{1, t}$ and $X_{2, t}$ are auxiliary variables representing $\mathrm{CO}_{2}$ concentration increases in shallow and lower oceans, respectively, also measured in GtC from 1750.

Temperature increase $H_{t}$ (degrees Celsius from 1900) develops according to

$$
\begin{aligned}
H_{t+1} & =\left(1-a_{0}\right) H_{t}+a_{1} \log \left(M_{t+1}\right)+a_{2} Z_{t}+F_{t+1}, \\
Z_{t+1} & =\left(1-a_{3}\right) Z_{t}+a_{3} H_{t},
\end{aligned}
$$

where $Z_{t}$ is an auxiliary variable representing the temperature increase of the lower oceans, also measured in degrees Celsius from 1900, and $F_{t+1}$ is exogenous radiative forcing.

In each period $t$, the fraction of GDP not spent on abatement or 'damage' is either consumed $\left(C_{t}\right)$ or invested $\left(I_{t}\right)$ along the budget constraint

$$
\left(1-\omega_{t}-\xi H_{t}^{2}\right) Y_{t}=C_{t}+I_{t}
$$

A fraction $\omega_{t}$ of $Y_{t}$ is spent on abatement, and we specify the abatement cost fraction as

$$
\omega_{t}=\psi_{t} \mu_{t}^{\theta} \quad(\theta>1) .
$$

When $\mu_{t}$ increases then so does $\omega_{t}$, and a larger fraction of GDP will be spent on abatement.

Damage is represented by a fraction $\xi H_{t}^{2}$ of $Y_{t}$ and it depends only on temperature. The optimal temperature is $H_{t}=0$, the temperature in 1900. Deviations from the optimal temperature cause damage. For very high and very low temperatures the fraction becomes large, but (given the value of $\xi$ ) it will still be a fraction between zero and one, unless in truly catastrophic cases.

As in $[20,21]$ one period is five years. Period 1 refers to the time interval 2015-2019, period 2 to 2020-2024, and so on. Stock variables are measured at the beginning of the period; for example, $K_{1}$ denotes capital in the year 2015 . We choose the exogenous variables such that $L_{t}>0, A_{t}>0, E_{t}^{0}>0, \sigma_{t}>0$, and $0<\psi_{t}<1$. The policy variables must satisfy

$$
C_{t} \geq 0, \quad I_{t} \geq 0, \quad \mu_{t} \geq 0 .
$$

Nordhaus $[21,22]$ allows negative-emission technologies by setting an upper bound on $\mu_{t}$ of 1.2 (rather than 1.0) from period 30 onwards (year 2160), which implies that emissions can become negative by (3), and in fact this upper bound is reached in the DICE output from period 46 onwards (year 2240). The idea of negative emissions is controversial. Anderson and Peters [2] state that negative-emission technologies are unjust and a high-stake gamble, while a recent editorial in Nature ([7]) discusses the enormous effort required to carry out such technologies - an effort which would lead to a deterioration of the environment.

Given a utility function $U$ we define welfare in period $t$ as

$$
W_{t}=L_{t} U\left(C_{t} / L_{t}\right) \text {. }
$$


The policy maker has a finite horizon and maximizes total discounted welfare

$$
W=\sum_{t=1}^{T} \frac{W_{t}}{(1+\rho)^{t}} \quad(0<\rho<1),
$$

where $\rho$ denotes the discount rate and $T=100$ (500 years). Letting $x$ denote per capita consumption, the utility function $U(x)$ is assumed to be defined and strictly concave for all $x>0$. There are many such functions, but a popular choice is

$$
U(x)=\frac{x^{1-\alpha}-1}{1-\alpha} \quad(\alpha>0),
$$

where $\alpha$ denotes the elasticity of marginal utility of consumption. This is the socalled power function. Many authors, including Nordhaus, select this function. In earlier versions of the DICE model, Nordhaus [18] chooses $\alpha=2$ in which case $U(x)=1-1 / x$. Also popular is $\alpha=1$ in which case $U(x)=\log (x)$; see $[15,28]$. In the 2016 version of the DICE model $\alpha=1.45$.

\section{Temperature}

The DICE model thus consists of the seven equations (1)-(7). Four of these, equations (1)-(3) and (7), are not controversial. In the next three sections we shall discuss the $\mathrm{CO}_{2}$ equation (4), the temperature equation (5), and the budget constraint (6).

We start with the temperature equations in (5), which we now write in matrix form as

$$
x_{t+1}=A x_{t}+a_{t+1}
$$

where

$$
A=\left(\begin{array}{cc}
1-a_{0} & a_{2} \\
a_{3} & 1-a_{3}
\end{array}\right)
$$

and

$$
x_{t}=\left(\begin{array}{c}
H_{t} \\
Z_{t}
\end{array}\right), \quad a_{t}=\left(\begin{array}{c}
a_{1} \log \left(M_{t}\right)+F_{t} \\
0
\end{array}\right) .
$$

The matrix $A$ has two eigenvalues given by

$$
1-\frac{1-\eta_{1}}{2} \pm \frac{1}{2} \sqrt{\left(1-\eta_{1}\right)^{2}-4 \eta_{2}}
$$

where $\eta_{1}=1-a_{0}-a_{3}=0.8468$ and $\eta_{2}=\left(a_{0}-a_{2}\right) a_{3}=0.0030$, so that the eigenvalues are 0.9771 and 0.8697 , respectively. The largest eigenvalue is thus close to one and it would be equal to one if (and only if) $\eta_{2}=0$.

We can 'difference out' the auxiliary variable $Z$ and this gives

$$
\begin{aligned}
H_{t+1}= & \left(1+\eta_{1}\right) H_{t}-\left(\eta_{1}+\eta_{2}\right) H_{t-1} \\
& +\eta_{3} \log \left(M_{t+1}\right)-\left(\eta_{3}-\eta_{4}\right) \log \left(M_{t}\right)+\eta_{0 t},
\end{aligned}
$$

where $\eta_{3}=a_{1}=0.5338, \eta_{4}=a_{1} a_{3}=0.0133$, and $\eta_{0 t}=F_{t+1}-\left(1-a_{3}\right) F_{t}$. Eq. (13) does not contain $Z$ but, compared to (5a), it contains an additional lag in both $H$ and $\log (M)$. Note that $(13)$ is not invariant to scaling in $M$. 
Letting $\Delta$ be the (backward) difference operator defined by $\Delta x_{t+1}=x_{t+1}-x_{t}$, we can write (13) alternatively as

$$
\Delta H_{t+1}=\eta_{1} \Delta H_{t}+\eta_{3} \Delta \log \left(M_{t+1}\right)+\eta_{0 t}^{*},
$$

where $\eta_{0 t}^{*}=-\eta_{2} H_{t-1}+\eta_{4} \log \left(M_{t}\right)+\eta_{0 t}$. This equation in first differences can in turn be integrated to

$$
H_{t+1}=\eta_{0}+\eta_{1} H_{t}+\eta_{3} \log \left(M_{t+1}\right)+\sum_{j=1}^{t} \eta_{0 j}^{*},
$$

where $\eta_{0}=-3.3291$ is an integration constant. Notice that $H_{t+1}$ in (14) depends on $H_{t}$ but that the effect of $H_{t-1}$ (through $\eta_{0 t}^{*}$ ) is negligible, which is another way of saying that the largest eigenvalue of the matrix $A$ in (12) is close to one. Both are caused by the fact that $\eta_{2}$ is small. We emphasize that (12), (13), and (14) are equivalent descriptions of the DICE temperature equations. No approximation has yet taken place.

Given the DICE parameter values, in particular the fact that $\eta_{2}$ and $\eta_{4}$ are small, the partial sums $\sum_{j} \eta_{0 j}^{*}$ are well approximated by a linear trend with slope 0.025. This implies that if we run a regression on the equation

$$
H_{t+1}=\eta_{0}^{*}+\eta_{1}^{*} H_{t}+\eta_{2}^{*} \log \left(M_{t+1}\right)+\eta_{3}^{*} t
$$

we will get a good fit. If we leave out the linear trend, then the estimate of $\eta_{1}^{*}$ increases somewhat and the estimate of $\eta_{2}^{*}$ decreases somewhat.

Table 1 Simplified temperature equations. In (a), we report estimated coefficients and standard errors from a regression based on (15). Results without a time trend are in (b).

\begin{tabular}{ccccccc}
\hline & constant & $H_{t}$ & $\log \left(M_{t+1}\right)$ & trend/10 & $s$ & $\max \left|Q_{t}\right|$ \\
\hline (a) & -3.6772 & 0.8707 & 0.5826 & 0.0064 & 0.0032 & $0.43 \%$ \\
& $(0.0511)$ & $(0.0014)$ & $(0.0076)$ & $(0.0003)$ & & \\
(b) -2.8672 & 0.8954 & 0.4622 & - & 0.0068 & $1.70 \%$ \\
& $(0.0561)$ & $(0.0012)$ & $(0.0082)$ & & & \\
\hline
\end{tabular}

More precisely, we obtain the results in Table 1, where we note that in all regressions, figures, and numerical experiments that follow, $H_{t}$ (and similarly $M_{t}$ and other variables) take the optimal values as obtained from the General Algebraic Modeling System (GAMS) routine which optimizes welfare (10) in the DICE system.

Under (a) we report the estimated coefficients and standard errors from a regression of $H_{t+1}$ on a constant, $H_{t}, \log \left(M_{t+1}\right)$, and a time trend, as in (15). The fit is very good. In particular, letting $e$ denote the vector of residuals and $\hat{H}_{t}$ the predicted value of $H_{t}$ from the regression, and defining the regression variance $s^{2}$ and the relative deviations $Q_{t}$ as

$$
s^{2}=e^{\prime} e /(n-k), \quad Q_{t}=100\left(\hat{H}_{t}-H_{t}\right) / H_{t},
$$




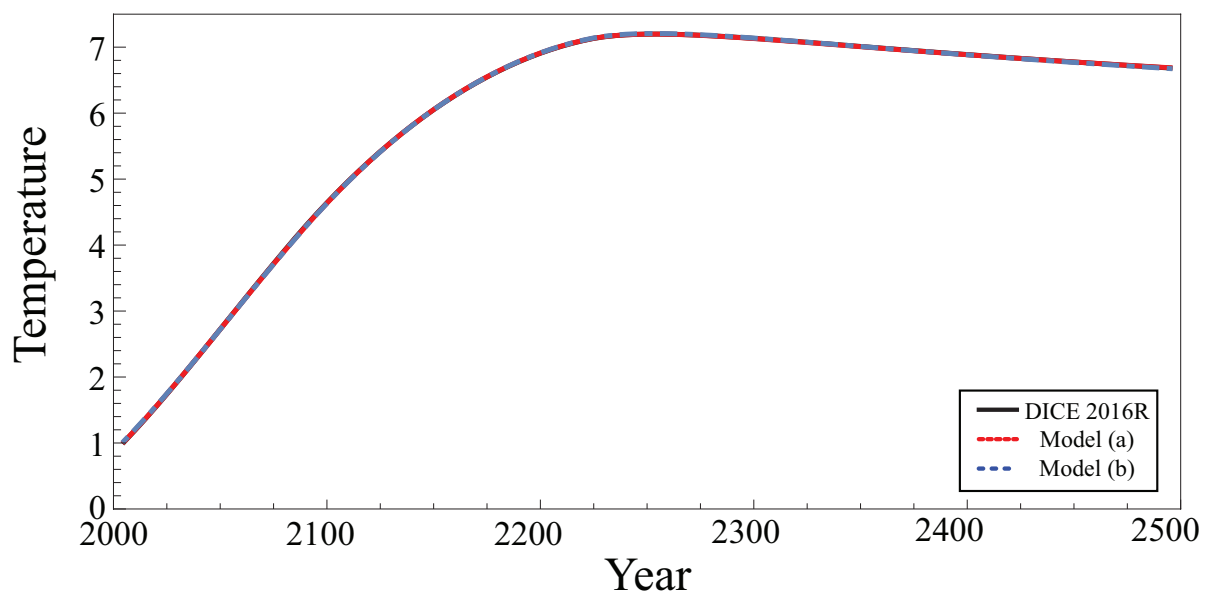

Figure 1 Temperature - time path for DICE 2016R and two simplified models. Model (a) is based on (15). Model (b) is based on (15) without a time trend.

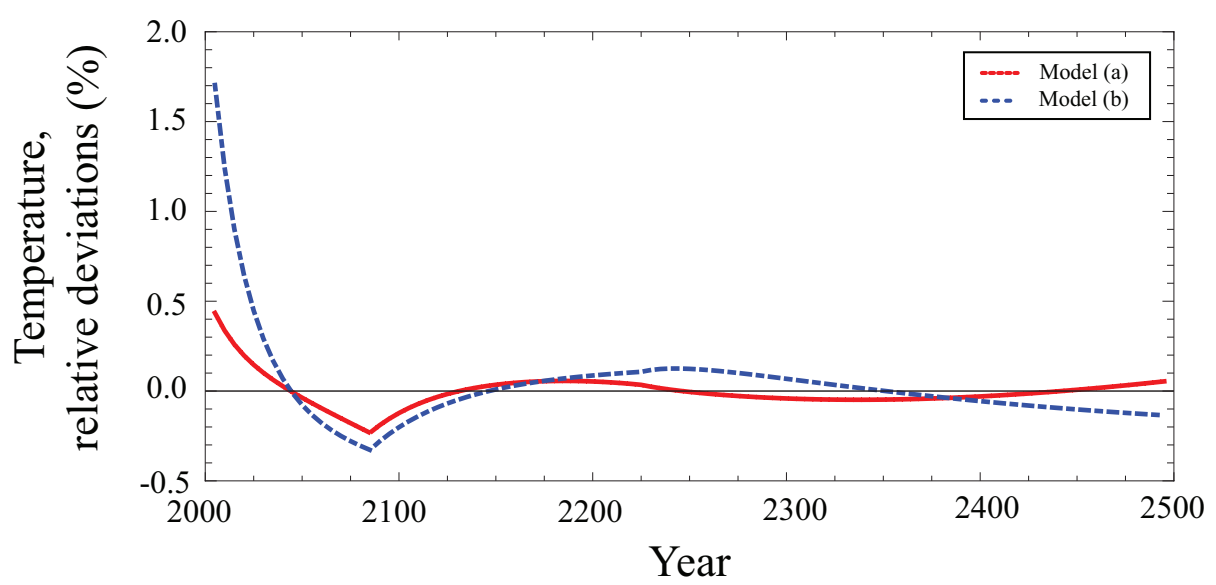

Figure 2 Temperature - Deviations (\%) of two simplified models relative to DICE $2016 \mathrm{R}$. Model (a) is based on (15). Model (b) is based on (15) without a time trend.

we find that $s=0.0032$ and $\max _{t}\left|Q_{t}\right|=0.43$ with $n=99$ and $k=4$. This shows that for a temperature increase of, say, 3 degrees Celsius the maximum error will be 0.013 degrees.

If we leave out the linear trend we obtain (b) which is almost as good, except that the error in the first few periods is somewhat higher. This is illustrated in Figures 1 and 2. In Figure 1 the time paths of temperature in DICE and the two models (a) and (b) are indistinguishable, reaching a maximum of 7.2 in 2270 . The relative deviations $Q_{t}$ are graphed in Figure 2. They are all below $0.5 \%$ except the first four periods in Model (b). Even though the estimated coefficient on the time trend is 'significant' it is not important, and the fit is essentially the same.

Summarizing, the simplified equation (15) provides a good approximation because (a) the coefficients in (13) correspond approximately to a first-order differ- 
ence equation; and (b) the omitted variable is essentially constant. The second approximation (without trend) is almost as good as the approximation in (15), and suffices for practical applications.

\section{$4 \mathrm{CO}_{2}$ concentration}

Next we consider the $\mathrm{CO}_{2}$ equations in (4), which we also write in matrix form as

$$
x_{t+1}=A x_{t}+a_{t},
$$

where now

$$
A=\left(\begin{array}{ccc}
1-b_{0} & b_{1} & 0 \\
b_{0} & 1-b_{1}-b_{3} & b_{2} \\
0 & b_{3} & 1-b_{2}
\end{array}\right)
$$

and

$$
x_{t}=\left(\begin{array}{c}
M_{t} \\
X_{1, t} \\
X_{2, t}
\end{array}\right), \quad a_{t}=\left(\begin{array}{c}
E_{t} \\
0 \\
0
\end{array}\right) .
$$

One of the three eigenvalues of $A$ equals one, and the two remaining eigenvalues are given by

$$
1-\frac{1-\phi_{1}}{2} \pm \frac{1}{2} \sqrt{\left(1-\phi_{1}\right)^{2}-4 \phi_{2}}
$$

where $\phi_{1}=1-b_{0}-b_{1}-b_{2}-b_{3}=0.675535$ and $\phi_{2}=b_{0} b_{2}+b_{0} b_{3}+b_{1} b_{2}=0.001303$, so that the two remaining eigenvalues take the values 0.995933 and 0.679602 , respectively. The largest eigenvalue is thus equal to one and the next eigenvalue is close to one; it would be equal to one if (and only if) $\phi_{2}=0$. This suggests that we should difference not once (as in the previous section) but twice, and this is precisely what we shall do.

As in the previous section we can 'difference out' the auxiliary variables $X_{1}$ and $X_{2}$, and this gives

$$
\begin{aligned}
M_{t+1}= & \left(\phi_{1}+2\right) M_{t}-\left(1+2 \phi_{1}+\phi_{2}\right) M_{t-1} \\
& +\left(\phi_{1}+\phi_{2}\right) M_{t-2}+E_{t}^{*}
\end{aligned}
$$

where

$$
E_{t}^{*}=E_{t}-\left(1+\lambda_{1}\right) E_{t-1}+\left(\lambda_{1}+\lambda_{2}\right) E_{t-2} .
$$

This equation does not contain $X_{1}$ and $X_{2}$ but it contains two additional lags in both $M$ and $E$. We can write (18) alternatively as

$$
\Delta M_{t+1}=\left(\phi_{1}+1\right) \Delta M_{t}-\left(\phi_{1}+\phi_{2}\right) \Delta M_{t-1}+E_{t}^{*},
$$

where we notice that there is no remainder term in $M_{t-2}$ because the largest eigenvalue of $A$ equals one exactly given the DICE parameters. This leads to

$$
M_{t+1}=\phi_{0}+\left(\phi_{1}+1\right) M_{t}-\left(\phi_{1}+\phi_{2}\right) M_{t-1}+E_{t}^{* *},
$$

where $E_{t}^{* *}=\sum_{j=1}^{t} E_{j}^{*}$ and $\phi_{0}=0.8761$ is an integration constant. This, in turn, can be written as

$$
\Delta M_{t+1}=\phi_{0}+\phi_{1} \Delta M_{t}-\phi_{2} M_{t-1}+E_{t}^{* *}
$$


so that

$$
M_{t+1}=\phi_{00}+\phi_{0} t+\phi_{1} M_{t}-\phi_{2} \sum_{j=1}^{t-1} M_{j}+\sum_{j=1}^{t} w_{t j} E_{j},
$$

where $\phi_{00}=263.2837$ is an integration constant and

$$
\begin{gathered}
\sum_{j=1}^{t} w_{t j} E_{j}=\sum_{j=1}^{t} E_{j}^{* *}=\sum_{j=1}^{t} \sum_{i=1}^{j} E_{i}^{*}=\sum_{j=1}^{t}(t-j+1) E_{j}^{*} \\
=E_{t}+\left(1-\lambda_{1}\right) \sum_{j=1}^{t-1} E_{j}+\lambda_{2} \sum_{j=1}^{t-2}(t-j-1) E_{j},
\end{gathered}
$$

so that $w_{t t}=1$ and

$$
w_{t j}=1-\lambda_{1}+(t-j-1) \lambda_{2} \quad(j=1, \ldots, t-1) .
$$

The DICE weights $w_{t j}$ are thus slightly increasing rather than decreasing, which is a little awkward. Notice that equations (17)-(20) are equivalent descriptions of the DICE $\mathrm{CO}_{2}$ equations. No approximation has yet taken place.

Since $\phi_{2}=0.0013$ and $\lambda_{2}=0.0003$ are close to zero, (20) will be well approximated by

$$
M_{t+1} \approx \phi_{00}+\phi_{0} t+\phi_{1} M_{t}+E_{t}+\left(1-\lambda_{1}\right) \sum_{j=1}^{t-1} E_{j} .
$$

In fact we will run regressions on the equation

$$
M_{t+1}=\phi_{0}^{*}+\phi_{1}^{*} M_{t}+\phi_{2}^{*} E_{t}+\phi_{3}^{*} t
$$

and simplifications thereof.

Table 2 Simplified $\mathrm{CO}_{2}$ equations. Panel (a) reports estimated coefficients and standard errors for an unrestricted regression based on (21). Panel (b) restricts the time trend to be zero. Panel (c) additionally restricts the constant term to be zero. Panel (d) additionally restricts the coefficient of $E_{t}$ to be one. The statistics $s$ and $Q_{t}$ are defined in (16).

\begin{tabular}{ccccccc}
\hline & constant & $M_{t}$ & $E_{t}$ & trend & $s$ & $\max \left|Q_{t}\right|$ \\
\hline (a) & 16.27 & 0.9900 & 0.6166 & 0.0317 & 1.52 & $0.35 \%$ \\
& $(1.08)$ & $(0.0004)$ & $(0.0067)$ & $(0.0098)$ & & \\
(b) & 17.94 & 0.9902 & 0.6001 & - & 1.59 & $0.39 \%$ \\
& $(0.99)$ & $(0.0004)$ & $(0.0046)$ & & & \\
(c) & - & 0.9975 & 0.6549 & - & 3.33 & $0.92 \%$ \\
& & $(0.0002)$ & $(0.0073)$ & - & 16.35 & $1.66 \%$ \\
(d) & - & 0.9942 & 1.0000 & - & & \\
& & $(0.0007)$ & $(-)$ & & & \\
\hline
\end{tabular}

This leads to the results in Table 2. Under (a) we regress $M_{t+1}$ on all four variables, under (b) we delete the trend, under (c) we also delete the constant term, and under (d) we restrict the coefficient of $E_{t}$ to be one. The last model is 


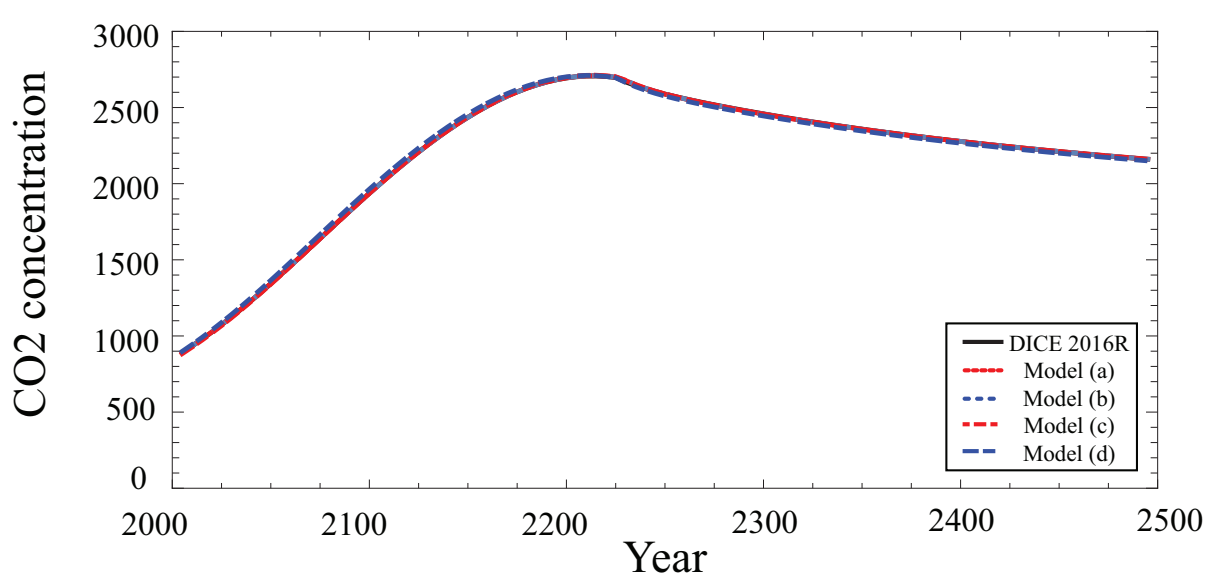

Figure $3 \mathrm{CO}_{2}$ concentration - time path for DICE 2016R and four simplified models. Model (a) is based on (21). Model (b) is based on (21) without a time trend. Model (c) is based on (21) without both a time trend and a constant term. Model (d) additionally restricts the coefficient of $E_{t}$ to be one.

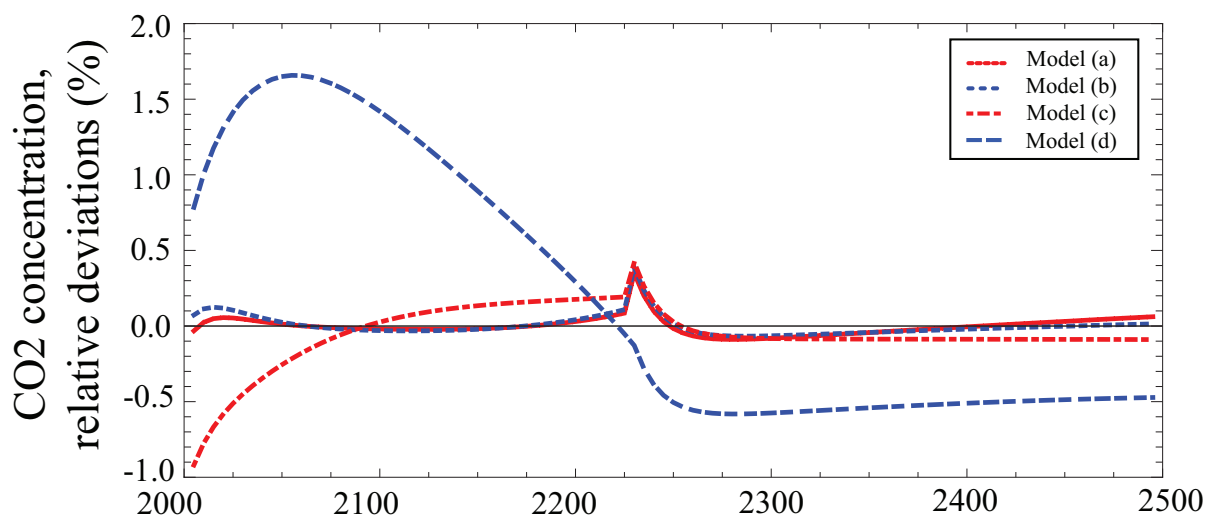

Figure $4 \mathrm{CO}_{2}$ concentration - Deviations (\%) of four simplified models relative to DICE 2016R. Model (a) is based on (21). Model (b) is based on (21) without a time trend. Model (c) is based on (21) without both a time trend and a constant term. Model (d) additionally restricts the coefficient of $E_{t}$ to be one.

the simplest and mirrors capital accumulation in (2). The fit is very good in all cases, as can be seen from the values of $s$ and $\max \left|Q_{t}\right|$, and also from Figures 3 and 4 .

In Figure 3 the time paths of $\mathrm{CO}_{2}$ of DICE and the four models (a)-(d) are indistinguishable, reaching a maximum of 2707 in 2230. The relative deviations $Q_{t}$ are graphed in Figure 4. In Models (a) and (b) the relative deviations are all below $0.4 \%$ in absolute value. In Model (c) the relative deviations are all below $0.6 \%$ in absolute value, except in the first three periods. In Model (d) the relative deviations are larger than $0.6 \%$ up to period 36 (year 2190) and smaller than $0.6 \%$ afterwards, with a maximum of $1.7 \%$ in period 12 (year 2070) where $M_{t}=1402$ (the DICE output) and $\hat{M}_{t}=1425$ (the predicted value of $M_{t}$ from the regression). 
The simplified equation (21) thus provides an excellent approximation to the DICE results because the coefficients in (18) correspond approximately to a secondorder difference equation. Model (b) is possibly the preferred approximation although the simplest Model (d) will suffice for most practical applications.

\section{Damage and abatement}

The damage-abatement function in DICE specifies two fractions, $\omega_{t}$ (abatement) and $\xi H_{t}^{2}$ (damage), of $Y_{t}$ which reduce $Y_{t}$ so that less money is available for investment and consumption along the budget constraint. In DICE this fraction is specified as

$$
1-\omega_{t}-\xi H_{t}^{2}
$$

For very high and very low temperatures the fraction becomes large, but (given the value of $\xi$ ) it will still be a fraction between zero and one, unless in truly catastrophic cases when $H_{t}>20.58$, that is, when the temperature in period $t$ is more than 20 degrees Celsius higher than in 1900. Of course, other forms of the damage function are possible; see $[28,32,1,19]$. Howard and Sterner [11] emphasize the importance of the damage function in accurately estimating coefficient and standard error bias.

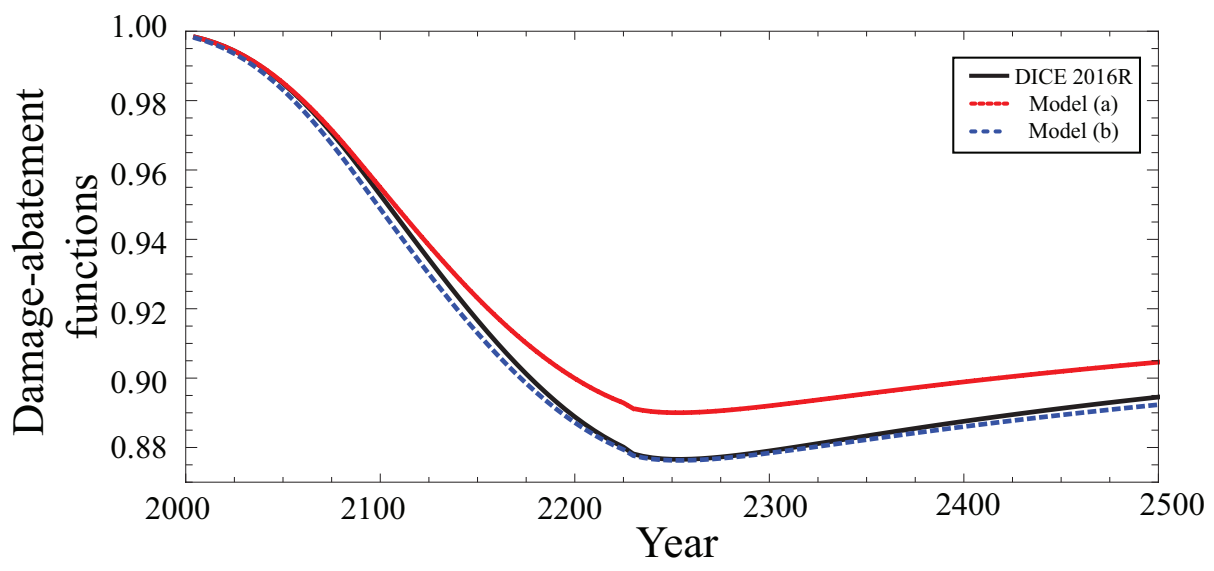

Figure 5 Damage-abatement functions. Model (a) uses the DICE value of $\xi$. Model (b) uses the optimal value of $\xi$.

In Figure 5 the graph labeled DICE 2016R contains the time path of this fraction. Models (a) and (b) use an alternative specification, namely

$$
\frac{1-\omega_{t}}{1+\xi H_{t}^{2}}
$$

based on the fact that the difference

$$
\left(1-\omega_{t}-\xi H_{t}^{2}\right)-\frac{1-\omega_{t}}{1+\xi H_{t}^{2}}=\frac{-\left(\omega_{t}+\xi H_{t}^{2}\right)\left(\xi H_{t}^{2}\right)}{1+\xi H_{t}^{2}}
$$


is small, about $1 \%$ in relative terms.

The alternative specification is of interest because we may wish to randomize $\xi$, as in [14]; see also [12,13]. This is difficult under the DICE specification because $1-\omega_{t}-\xi H_{t}^{2}$ could become negative (under extreme circumstances), while the alternative specification is always positive provided $\xi>0$.

In Model (a) we use the same value for $\xi$ as in the DICE model, while in Model (b) we use an 'optimal' value $\xi^{*}=0.00265$ which brings the lines closer together; in fact, DICE and Model (b) are indistinguishable in the figure. The value $\xi^{*}$ is obtained by minimizing the sum of squares

$$
\sum_{t=1}^{T}\left(1-\omega_{t}-\xi H_{t}^{2}-\frac{1-\omega_{t}}{1+\xi^{*} H_{t}^{2}}\right)^{2} .
$$

with respect to $\xi^{*}$. In summary, for a suitable choice of $\xi$ we obtain an alternative for the DICE damage function which lends itself better to studying situations of uncertainty or catastrophe.

\section{The S-DICE model}

We summarize our proposed S-DICE (simplified DICE) model with the relevant parameters. The S-DICE model is the DICE model, but with the temperature equation replaced by the new (simplest) temperature equation

$$
H_{t+1}=\eta_{0}^{*}+\eta_{1}^{*} H_{t}+\eta_{2}^{*} \log \left(M_{t+1}\right)
$$

with

$$
\eta_{0}^{*}=-2.8672, \quad \eta_{1}^{*}=0.8954, \quad \eta_{2}^{*}=0.4622,
$$

and the $\mathrm{CO}_{2}$ equation replaced by

$$
M_{t+1}=\phi_{1}^{*} M_{t}+E_{t}, \quad \phi_{1}^{*}=0.9942 .
$$

For the damage-abatement equation we propose

$$
\frac{1-\omega_{t}}{1+\xi^{*} H_{t}^{2}}, \quad \xi^{*}=0.00265
$$

instead of the DICE specification

$$
1-\omega_{t}-\xi H_{t}^{2}, \quad \xi=0.00236 .
$$

In addition, one may wish to set $\mu \leq 1.0$ instead of the upper bound $\mu \leq 1.2$ as used in DICE. Thus, there are four differences between DICE and S-DICE.

So far we have worked within the framework of the optimized DICE model (to be precise, the baseline version with ifopt $=0$ ), so that variables such as $H_{t}$ and $M_{t}$ take their optimal values as obtained from the DICE GAMS routine, as emphasized in Sect. 3.

To judge how the S-DICE model compares to DICE we should optimize SDICE itself. In Table 3 we compare three scenarios: DICE and two scenarios of S-DICE, namely (a) where we use the new temperature equation (22) and the $\mathrm{CO}_{2}$ equation (23), but not the new damage-abatement equation, while also keeping 
Table 3 DICE versus S-DICE (a) and (b). Model (a) uses the DICE value of $\xi$. Model (b) uses the optimal value of $\xi$.

\begin{tabular}{|c|c|c|c|c|c|c|c|c|}
\hline & \multicolumn{5}{|c|}{ Short term } & \multicolumn{3}{|c|}{ Longer term } \\
\hline & 2020 & 2030 & 2040 & 2050 & 2060 & 2100 & 2150 & 2200 \\
\hline \multicolumn{9}{|c|}{ Temperature $H$} \\
\hline DICE & 1.0 & 1.4 & 1.7 & 2.1 & 2.5 & 4.1 & 5.7 & 6.7 \\
\hline (a) & 1.0 & 1.4 & 1.8 & 2.3 & 2.7 & 4.5 & 6.4 & 7.6 \\
\hline (b) & 1.0 & 1.4 & 1.8 & 2.1 & 2.5 & 3.6 & 4.1 & 4.1 \\
\hline \multicolumn{9}{|c|}{ Capital K } \\
\hline DICE & 268 & 375 & 505 & 660 & 840 & 1830 & 3691 & 6528 \\
\hline (a) & 267 & 373 & 502 & 655 & 833 & 1802 & 3583 & 5968 \\
\hline (b) & 268 & 374 & 504 & 660 & 842 & 1857 & 3884 & 6871 \\
\hline \multicolumn{9}{|c|}{$\mathrm{CO}_{2}$ concentration $M$} \\
\hline DICE & 891 & 978 & 1073 & 1177 & 1287 & 1760 & 2306 & 2649 \\
\hline (a) & 898 & 1006 & 1129 & 1266 & 1416 & 2078 & 2859 & 3346 \\
\hline (b) & 889 & 972 & 1059 & 1145 & 1226 & 1379 & 1304 & 1231 \\
\hline \multicolumn{9}{|c|}{ Abatement fraction $\mu$} \\
\hline DICE & 0.03 & 0.04 & 0.04 & 0.05 & 0.06 & 0.11 & 0.24 & 0.53 \\
\hline (a) & 0.03 & 0.04 & 0.04 & 0.05 & 0.06 & 0.11 & 0.24 & 0.53 \\
\hline (b) & 0.23 & 0.29 & 0.36 & 0.44 & 0.54 & 1.00 & 1.00 & 1.00 \\
\hline \multicolumn{9}{|c|}{ Consumption $C$} \\
\hline DICE & 465 & 643 & 857 & 1105 & 1390 & 2881 & 5494 & 8860 \\
\hline (a) & 465 & 643 & 855 & 1103 & 1385 & 2847 & 5335 & 8433 \\
\hline (b) & 464 & 641 & 851 & 1096 & 1376 & 2854 & 5718 & 9718 \\
\hline \multicolumn{9}{|c|}{ Social cost of carbon $S C C$} \\
\hline $\mathrm{DICE}$ & 37 & 52 & 69 & 90 & 115 & 255 & 525 & 915 \\
\hline (a) & 50 & 68 & 90 & 116 & 146 & 313 & 633 & 1089 \\
\hline (b) & 53 & 72 & 96 & 124 & 159 & 363 & 814 & 1488 \\
\hline
\end{tabular}

the 1.2 bound for $\mu$ as in DICE; and (b) where we use the full S-DICE model with all four changes. We present these results for the short term (up to 40 years) and the longer term (100-200 years).

The most important conclusion from Table 3 is that the difference between DICE and version (a) of S-DICE is small. This is true for the short and 'longer' term (up to 200 years) and remains true for the very long term (up to 500 years), as the three figures below will demonstrate. Hence, the idea that simplifications of DICE tend to have poor long-term properties is not true, at least when we consider simplifications of the temperature and $\mathrm{CO}_{2}$ equations, which is the main topic of the current paper. In fact, the comparisons we report here give an upper bound on the approximation error of S-DICE to DICE: we could have further reduced the approximation error by calibrating the S-DICE parameters so that optimal S-DICE is as close as possible to optimal DICE.

When we add two further changes, namely the damage-abatement function and the upper bound on $\mu$, then we obtain version (b) of S-DICE. Here the $\mathrm{CO}_{2}$ concentration and hence the temperature is much lower than in DICE and SDICE(a), because the new damage-abatement function of scenario (b) leads to a 
rapid increase in $\mu$. In order to better understand the reason behind this difference we study below not only versions (a) and (b) but also the two intermediate versions where only one of the additional changes is implemented.

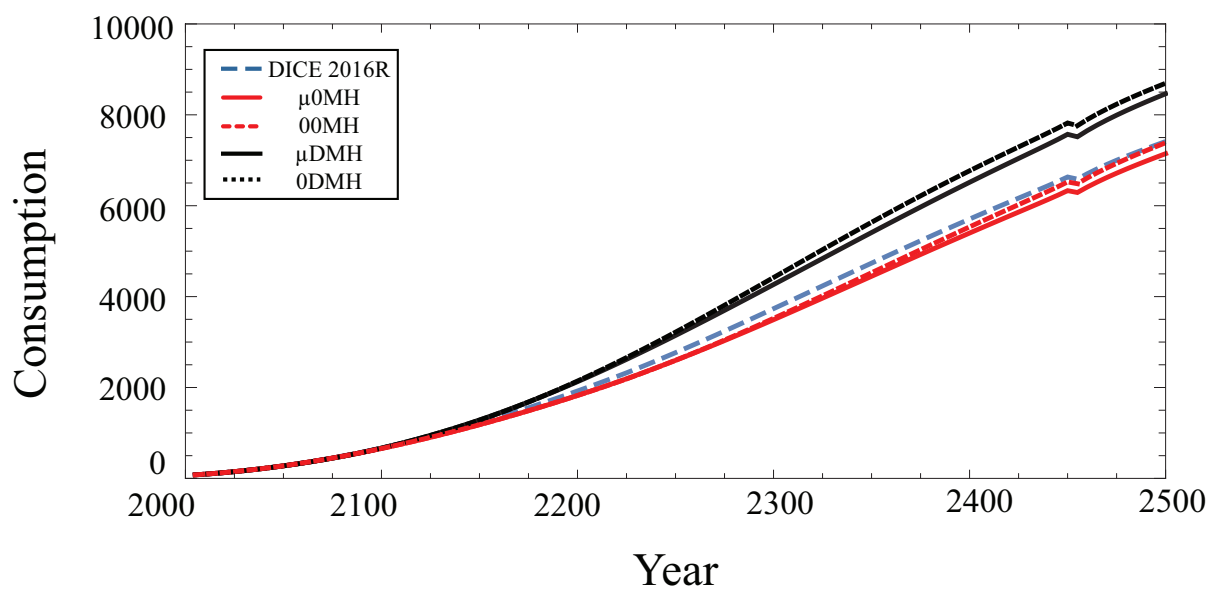

Figure 6 Consumption - DICE and S-DICE compared. Refer to the main text for the meaning of scenario acronyms such as $\mu D M H$.

We denote by $\mu D M H$ the scenario where all four changes have been implemented: $\mu$ for the 1.0 upper bound on $\mu, D$ for the damage-abatement function, $M$ for the $\mathrm{CO}_{2}$ concentration equation, and $H$ for the temperature equation. A zero indicates that this change has not been implemented. Hence, DICE is 0000, model (a) is $00 M H$ and model (b) is $\mu D M H$. To these three scenarios we now add the intermediate scenarios $\mu O M H$ and $O D M H$.

In Fig. 6 we present the time paths of consumption of the five scenarios. Consumption (and, similarly, capital accumulation) is not much affected by the different scenarios. The simplified model $00 M H$ with the original damage-abatement function and constraint on $\mu$ yields similar time paths to DICE, and even the full $\mathrm{S}-\mathrm{DICE}$ model $\mu D M H$ remains close to the DICE results, even in the long run. The consumption paths with a positive emissions constraint are lower than the paths without the constraint. This is because the resource allocation to abatement is larger when negative emissions are not allowed.

In the full S-DICE scenario, the new damage-abatement function causes a rapid reduction of $\mathrm{CO}_{2}$ in the short term. In fact, $\mu$ reaches its maximum $(\mu=1)$ in 2100 when $\mathrm{CO}_{2}$ concentration, and hence temperature, reaches its highest value. After $2100, \mathrm{CO}_{2}$ concentration and temperature gradually decline. Although $\mu$ reaches its maximum, consumption becomes larger than under the original damage-abatement function, due to smaller damage.

In Fig. 7 we present the time paths of temperature. With the old damageabatement function, the time paths are close. Hence the different behavior is not caused by the constraint on $\mu$ but by the form of the damage-abatement fraction. If we include the new damage-abatement fraction but keep the constraint on $\mu$ at $1.2(O D M H)$, so that we allow negative emissions, then the difference with 


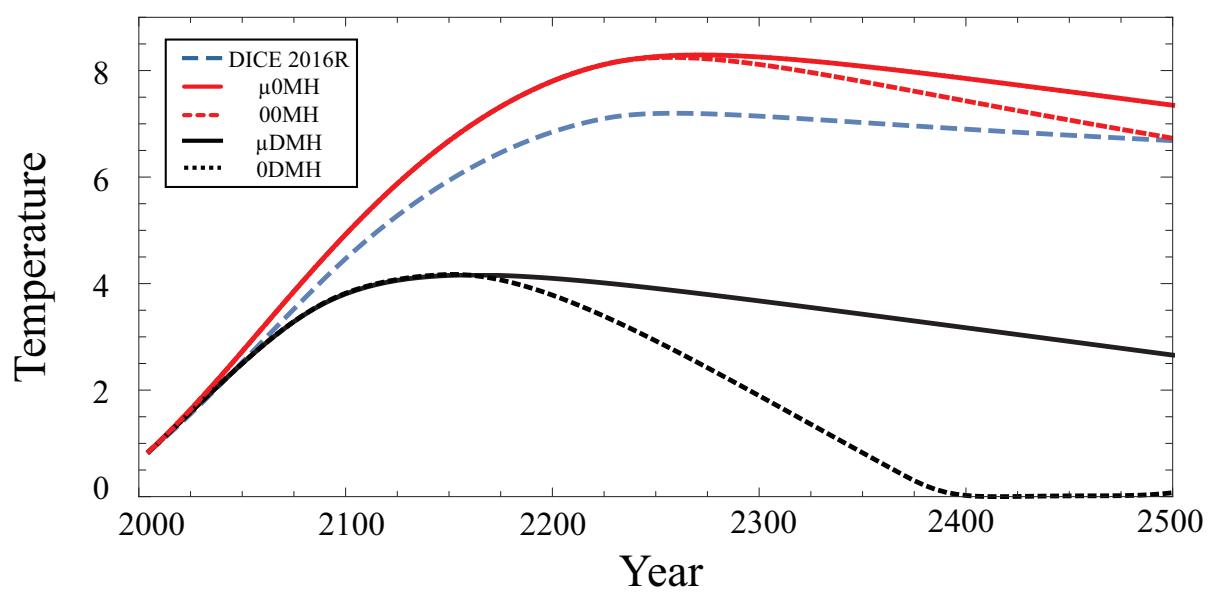

Figure 7 Temperature - DICE and S-DICE compared. Refer to the main text for the meaning of scenario acronyms such as $\mu D M H$.

DICE is much larger than if we include all four changes $(\mu D M H)$. When the modified damage-abatement function and the constraint of positive emissions are both used, then the positivity constraint on temperature increase imposed by Nordhaus' GAMS code is binding from the year 2400 onwards, which affects emissions and causes a higher abatement rate and a lower level of $\mathrm{CO}_{2}$ emissions in the short term. The figure for $\mathrm{CO}_{2}$ concentration is similar to temperature.

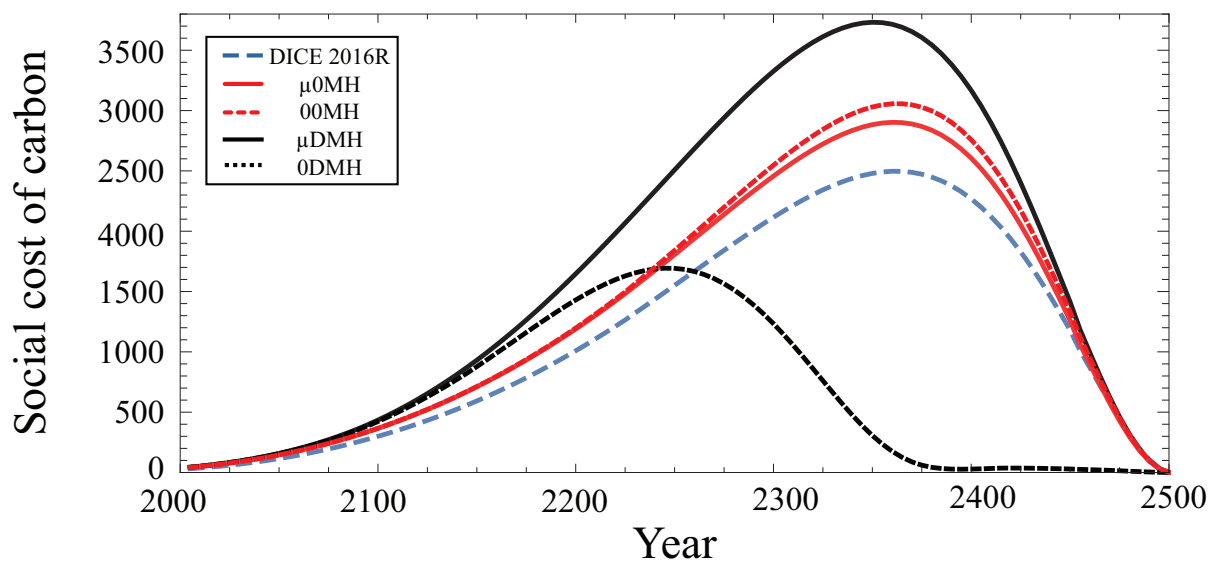

Figure 8 Social cost of carbon - DICE and S-DICE compared. Refer to the main text for the meaning of scenario acronyms such as $\mu D M H$.

Finally, we present the social cost of carbon (SCC), which attempts to answer the question how much we should be willing to pay to avert future climate damages. More precisely, the SCC tries to add up all the quantifiable costs and benefits of emitting one additional tonne of $\mathrm{CO}_{2}$. This value can then be used to weigh the benefits of reduced warming against the costs of cutting emissions. 
In Fig. 8 we present the SCC for the same five scenarios as before. We see that when negative emissions are allowed the social cost of carbon is lower than when negative emissions are not allowed. With the original damage-abatement function and the simplified temperature and $\mathrm{CO}_{2}$ equations, the SCC paths are similar to DICE, but the new damage-abatement function leads to a higher SCC in the short run so as to reduce $\mathrm{CO}_{2}$ rapidly. Thus, when negative emissions are not allowed, the social cost of carbon becomes much higher.

From the tables and figures we draw the following general conclusions. First, replacing the temperature and $\mathrm{CO}_{2}$ equations by two simpler, more transparent, more robust, and easier to interpret equations does not much affect the DICE output, not even in the long run. Second, the optimized results are sensitive to the form of the damage-abatement function, especially if we allow for negative emissions. Third, the full S-DICE model leads to optimized values that are different from DICE, but within the bounds of reason. After all, there is no reason to believe that the DICE model is the truth. If S-DICE deviates from DICE then this does not necessarily mean that S-DICE is further from the truth than DICE or is less useful as a policy instrument. As noted in the introduction, there is value in simplicity. Models should be, as Einstein put it, 'as simple as possible but not simpler'.

Acknowledgements We are grateful to the editor and advisory editor, and to Peter Boswijk, Rick van der Ploeg, and Hiroaki Sakamoto for comments and suggestions.

\section{Declarations}

\subsection{Funding}

This research was funded in part by the Japan Society for the Promotion of Science (JSPS), KAKENHI Grant JP19K01669 (Ikefuji), and the Netherlands Organization for Scientific Research (NWO) under grant Vidi-2009 (Laeven).

\subsection{Conflict of interest}

The authors have no relevant financial or non-financial interests to disclose.

7.3 Availability of data and material

Not applicable.

\subsection{Code availability}

Code is available upon request. 


\section{Appendix}

In this Appendix we present two tables which together contain all variable and parameter definitions required to compute the optimum in DICE (the Nordhaus model) and S-DICE (our simplified version of DICE): the variables employed in S-DICE and DICE and their relationship, and the initial values of the six state variables of DICE (Table 4); and the parameters employed in S-DICE and DICE and their relationship (Table 5).

Table 4 Variables in S-DICE and DICE

\begin{tabular}{|c|c|c|}
\hline S-DICE & DICE & Initial values \\
\hline \multicolumn{3}{|c|}{ State variables } \\
\hline$H_{t}$ & $\operatorname{TATM}(\mathrm{t})$ & $H_{1}=0.85$ \\
\hline$K_{t}$ & $K(t)$ & $K_{1}=223$ \\
\hline$M_{t}$ & $\operatorname{MAT}(\mathrm{t})$ & $M_{1}=851$ \\
\hline \multicolumn{3}{|c|}{ Policy variables } \\
\hline$I_{t}$ & tstep $\times \mathrm{I}(\mathrm{t})$ & \\
\hline$\mu_{t}$ & $\mathrm{MIU}(\mathrm{t})$ & \\
\hline$C_{t}$ & tstep $\times \mathrm{C}(\mathrm{t})$ & \\
\hline \multicolumn{3}{|c|}{ Exogenous variables } \\
\hline$A_{t}$ & tstep $\times \mathrm{AL}(\mathrm{t})$ & \\
\hline$E_{t}^{0}$ & tstep $\times \operatorname{ETREE}(\mathrm{t}) / 3.666$ & \\
\hline$F_{t}$ & $-a_{1} \log (588)+0.1005 \times$ FORCOTH $(\mathrm{t})$ & \\
\hline$L_{t}$ & $\mathrm{~L}(\mathrm{t}) / 1000$ & \\
\hline$\psi_{t}$ & $\operatorname{COST} 1(\mathrm{t})$ & \\
\hline$\sigma_{t}$ & $\operatorname{SIGMA}(\mathrm{t}) / 3.666$ & \\
\hline \multicolumn{3}{|c|}{ Auxiliary variables } \\
\hline$E_{t}$ & tstep $\times \mathrm{E}(\mathrm{t}) / 3.666$ & \\
\hline$Y_{t}$ & tstep $\times$ YGROSS $(\mathrm{t})$ & \\
\hline$\omega_{t}$ & defined in (7) & \\
\hline$X_{1, t}$ & $\mathrm{MU}(\mathrm{t})$ & $X_{1,1}=460$ \\
\hline$X_{2, t}$ & $\operatorname{ML}(\mathrm{t})$ & $X_{2,1}=1740$ \\
\hline$Z_{t}$ & TOCEAN $(\mathrm{t})$ & $Z_{1}=0.0068$ \\
\hline
\end{tabular}


Table 5 Parameters in S-DICE and DICE

\begin{tabular}{|c|c|c|c|}
\hline S-DICE & DICE & Value & Description \\
\hline \multicolumn{4}{|c|}{ Basic parameters } \\
\hline & $a 1$ & 0 & \\
\hline \multirow[t]{4}{*}{$\xi$} & a2 & 0.00236 & \\
\hline & a3 & 2 & \\
\hline & tstep & 5 & \\
\hline & $d k$ & 0.1 & \\
\hline \multirow[t]{2}{*}{$\delta$} & & 0.40951 & $1-\delta=(1-d k)^{t s t e p}$ \\
\hline & prstp & 0.015 & \\
\hline$\rho$ & & 0.077284 & $1+\rho=(1+\text { prstp })^{t s t e p}$ \\
\hline$\theta$ & expcost2 & 2.6 & \\
\hline$\gamma$ & gama & 0.3 & \\
\hline$\alpha$ & elasmu & 1.45 & \\
\hline$a_{0}$ & & 0.128189 & $a_{0}=(c 1)($ fco22 $x) /($ t2xco2 $)+(c 1)(c 3)$ \\
\hline$a_{1}$ & & 0.533755 & $a_{1}=(c 1)(f \operatorname{co} 22 x) / \log 2$ \\
\hline$a_{2}$ & $c 1 \times c 3$ & 0.008844 & \\
\hline$a_{3}$ & $c 4$ & 0.025 & \\
\hline$b_{0}$ & b12 & 0.12 & \\
\hline$b_{1}$ & b21 & 0.196 & \\
\hline$b_{2}$ & b32 & 0.001465 & \\
\hline$b_{3}$ & b23 & 0.007 & \\
\hline \multicolumn{4}{|c|}{ Additional parameters } \\
\hline$\eta_{0}$ & & -3.329093 & \\
\hline$\eta_{1}$ & & 0.846811 & $\eta_{1}=1-a_{0}-a_{3}$ \\
\hline$\eta_{2}$ & & 0.002984 & $\eta_{2}=\left(a_{0}-a_{2}\right) a_{3}$ \\
\hline$\eta_{3}$ & & 0.533755 & $\eta_{3}=a_{1}$ \\
\hline$\eta_{4}$ & & 0.013344 & $\eta_{4}=a_{1} a_{3}$ \\
\hline$\phi_{0}$ & & 0.876134 & \\
\hline$\phi_{00}$ & & 263.283680 & \\
\hline$\phi_{1}$ & & 0.675535 & $\phi_{1}=1-b_{0}-b_{1}-b_{2}-b_{3}$ \\
\hline$\phi_{2}$ & & 0.001303 & $\phi_{2}=b_{0} b_{2}+b_{0} b_{3}+b_{1} b_{2}$ \\
\hline$\lambda_{1}$ & & 0.795535 & $\lambda_{1}=1-b_{1}-b_{2}-b_{3}$ \\
\hline$\lambda_{2}$ & & 0.000287 & $\lambda_{2}=b_{1} b_{2}$ \\
\hline
\end{tabular}




\section{References}

1. Ackerman, F., Stanton, E. A., Bueno, R. (2010). Fat tails, exponents, extreme uncertainty: Simulating catastrophe in DICE. Ecological Economics, 69, 1657-1665.

2. Anderson, K., Peters, G. (2016). The trouble with negative emissions. Science, 354, 14 October, 182-183.

3. Calel, R., Stainforth, D.A. (2017). On the physics of three integrated assessment models. Bulletin of the American Meteorological Society, 98, 1199-1216.

4. De Luca, G., Magnus, J. R., Peracchi, F. (2018). Balanced variable addition in linear models. Journal of Economic Surveys, 32, 1183-1200.

5. Dietz, S., Venmans, F. (2018). Cumulative carbon emissions and economic policy: In search of general principles. Centre for Climate Change Economics and Policy, Working Paper 317.

6. Editorial (2015). IAM helpful or not? Nature Climate Change, 5, 81.

7. Editorial (2018). Why current negative-emissions strategies remain 'magical thinking'. $N a$ ture, 554, 21 February, doi: 10.1038/d41586-018-02184-x

8. Gerlagh, R., Liski, M. (2018a). Consistent climate policies. Journal of the European Economic Association, 16, 1-44.

9. Gerlagh, R., Liski, M. (2018b). Carbon prices for the next hundred years. The Economic Journal, 128, 728-757.

10. Golosov, M., Hassler, J., Krusell, P., Tsyvinski, A. (2014). Optimal taxes on fossil fuel in general equilibrium. Econometrica, 82, 41-88

11. Howard, P. H., Sterner, T. (2017). Few and not so far between: A meta-analysis of climate damage estimates. Environmental Resource Economics, 68, 197-225.

12. Ikefuji, M., Laeven, R. J. A., Magnus, J. R., Muris, C. (2013). Pareto utility, Theory and Decision, 75, 43-57.

13. Ikefuji, M., Laeven, R. J. A., Magnus, J. R., Muris, C. (2015). Expected utility and catastrophic consumption risk, Insurance: Mathematics and Economics, 64, 306-312.

14. Ikefuji, M., Laeven, R. J. A., Magnus, J. R., Muris, C. (2020). Expected utility and catastrophic risk in a stochastic economy-climate model, Journal of Econometrics, 214, 110-129.

15. Kelly, D. L., Kolstad, C. D. (1999). Bayesian learning, growth, and pollution. Journal of Economic Dynamics \& Control, 23, 491-518.

16. Lemoine, D., Rudik, I. (2017). Steering the climate system: Using inertia to lower the cost of policy. American Economic Review, 107, 2947-2957.

17. Nordhaus, W. D. (1992). An optimal transition path for controlling greenhouse gases. Science, 258, 1315-1319.

18. Nordhaus, W. D. (2008) A question of balance: Weighing the options on global warming policies. Yale University Press, New Haven, CT.

19. Nordhaus W. D. (2013). The climate casino: Risk, uncertainty, and economics for a warming world. Yale University Press, New Haven, CT.

20. Nordhaus, W. D. (2017). Revisiting the social cost of carbon. Proceedings of the $U$. S. National Academy of Sciences, 114, 1518-1523, February 14.

21. Nordhaus, W. D. (2018a). Projections and uncertainties about climate change in an era of minimal climate policies. American Economic Journal: Economic Policy, 10, 333-360.

22. Nordhaus, W. D. (2018b). Evolution of modeling of the economics of global warming: Changes in the DICE model, 1992-2017. Climatic Change, 148, 623-640.

23. Pindyck, R. S. (2013). Climate change policy: What do the models tell us? Journal of Economic Literature, 51, 860-872.

24. Pindyck. R. S. (2017). The use and misuse of models for climate policy. Review of Environmental Economics and Policy, 11, 100-114.

25. Rezai, A., Van der Ploeg, F. (2016). Intergenerational inequality aversion, growth, and the role of damages: Occam's rule for the global carbon tax. Journal of the Association of Environmental and Resource Economists, 3, 493-522.

26. Ricke, K. L., Caldeira, K. (2014). Maximum warming occurs about one decade after a carbon dioxide emission. Environmental Research Letters, 9, 124002.

27. Roe, G. H, Bauman, Y. (2013). Climate sensitivity: Should the climate tail wag the policy dog? Climatic Change, 117, 647-662.

28. Stern, N. (2007). The economics of climate change: The Stern review. Cambridge University Press, Cambridge, UK.

29. Traeger, C. P. (2015). Analytic integrated assessment and uncertainty. Mimeo.

30. Van den Bijgaart, I., Gerlagh, R., Liski, M. (2016). A simple formula for the social cost of carbon. Journal of Environmental Economics and Management, 77, 75-94. 
31. Van der Ploeg, F. (2018). The safe carbon budget. Climatic Change, 147, 47-59.

32. Weitzman, M. L. (2009). On modeling and interpreting the economics of catastrophic climate change. The Review of Economics and Statistics, 91, 1-19. 